\title{
Association between e-cigarette use and parents' report of attention deficit hyperactivity disorder among US youth
}

\author{
Bekir Kaplan', Arik V. Marcell2,3, Tugba Kaplan', Joanna E. Cohen ${ }^{1}$
}

\begin{abstract}
INTRODUCTION There is paucity of literature that evaluates e-cigarette use rates among the youth with attention deficit hyperactivity disorder (ADHD). The aim of this study is to compare the rates of cigarette only, e-cigarette only, dual use, and initiation age of regular use and trying to quit cigarettes or e-cigarettes among the youth with and without ADHD.

Methods We used Population Assessment of Tobacco and Health (PATH) study Wave 3 (2015-2016) youth data, a nationally representative cross-sectional study in the US. The main outcome was tobacco use status of youth and ADHD diagnosis was based on parent report.

RESULTS The survey included 11801 youth (50\%, 12-14 years; 49\% female). Compared to youth without ADHD, the relative risk ratio (RRR) was 1.79 (95\% CI: 1.02-3.21) for cigarette only use, 1.41 (95\% CI: 1.01-2.21) for e-cigarette only use, 3.40 (95\% CI: 1.69-6.84) for dual use, 1.75 (95\% CI: 0.92-3.35) for cigarette and other product(s) use, 1.48 (95\% CI: 0.58-3.77) for e-cigarette and other product(s) use, and 3.37 (95\% CI: 1.88-6.17) for poly use among youth with ADHD, after adjusting for age group, sex, and race/ethnicity.

Conclusions Cigarette only use, e-cigarette only use, dual use of cigarettes and e-cigarettes, and poly use of cigarettes, e-cigarettes, and other product(s) were significantly associated with parent report of an ADHD diagnosis. It is critical for healthcare providers to be screening youth for e-cigarette use, especially youth who are diagnosed with ADHD.
\end{abstract}

\author{
AFFILIATION \\ 1 Institute for Global Tobacco \\ Control, Johns Hopkins \\ Bloomberg School of Public \\ Health, Baltimore, United \\ States \\ 2 Department of Pediatrics, \\ Johns Hopkins Medicine, \\ Baltimore, United States \\ 3 Population, Family and \\ Reproductive Health, Johns \\ Hopkins Bloomberg School \\ of Public Health, Baltimore, \\ United States \\ 4 Department of Medicine, \\ Anne Arundel Medical Center, \\ Annapolis, United States
}

CORRESPONDENCE TO Bekir Kaplan. Institute for Global Tobacco Control, Johns Hopkins Bloomberg School of Public Health, 2213 McElderry Street Fourth Floor, Baltimore, MD 21205, United States. E-mail: bkaplan9@jhu.edu

KEYWORDS

adolescent, electronic nicotine delivery product, attention deficit disorders

Received: 17 January 2021 Revised: 5 April 2021

Accepted: 22 April 2021

\section{INTRODUCTION}

E-cigarette use in the US has increased dramatically and exceeded use of combustible cigarettes among youth in recent years ${ }^{1}$. In the US, $19.6 \%$ of high school students (3.02 million) and $4.7 \%$ of middle school students ( 0.55 million) currently used e-cigarettes every day or somedays in $2020^{2}$. Most e-cigarettes contain nicotine and results from prior cross-sectional analysis show that e-cigarette use is associated with increased risk for cigarette initiation ${ }^{3}$. Past longitudinal and cross-sectional research shows that people with mental health conditions (MHC) are more likely to smoke cigarettes compared to those

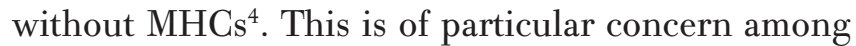
youth with attention deficit hyperactivity disorder (ADHD) - one of the most prevalent mental health disorders of childhood ${ }^{5,6}$ - since these youth may selfmedicate with nicotine products ${ }^{7}$ to regulate negative affect associated with attentional dysfunction and improve their attention 8

It has been well documented that adolescents with ADHD smoke more cigarettes compared to those 
without $\mathrm{ADHD}^{9-13}$. Moreover, earlier initiation of cigarette smoking ${ }^{14}$, quicker progression to regular ${\text { cigarette } \text { use }^{15} \text {, more severe nicotine dependence }}^{9}$, and more difficulty quitting smoking ${ }^{16}$ have been reported among people with ADHD. In addition, early cigarette use may increase the likelihood for subsequent substance use disorders in ADHD individuals ${ }^{17}$. To date, very little research has assessed the association between ADHD and e-cigarette use among youth ${ }^{18,19}$. In a one-year longitudinal study of ninth grade students in California, ADHD symptomology was associated with increased risk of e-cigarette initiation ${ }^{18}$. Another longitudinal study of high school seniors from four high schools in the US mid-Atlantic region reported ADHD symptoms in high school predicted increased risk for e-cigarette use in college $^{19}$. To our knowledge, the association between ADHD and e-cigarette use among youth has not been evaluated using nationally representative US data. This is especially relevant since e-cigarette use in the US has increased dramatically and exceeded use of combustible cigarettes among youth in recent years ${ }^{1}$ and nearly $40 \%$ of all adolescents have low levels of ADHD symptomatology ${ }^{5,6}$. Exploring the association between ADHD and e-cigarette initiation age, and e-cigarette quit attempts may help to understand the increase in e-cigarette use among youth.

To address gaps in the current literature, the main aim of this study was to compare the rates of cigarette use only, e-cigarette use only, and dual use of cigarettes and e-cigarette, among youth (aged 1217 years) with and without ADHD, using nationally representative data. We also explored the initiation age of regular use and trying to quit cigarettes or e-cigarettes among youth with and without an ADHD diagnosis. Such analyses are critical given that childhood externalizing disorders, primarily ADHD, have been defined as strongly predictive of nicotine use and dependence in the National Comorbidity Survey Replication ${ }^{20}$.

\section{METHODS}

\section{Study procedures}

This study uses publicly available youth data from wave 3 (October 2015-2016) of the Population Assessment of Tobacco and Health (PATH) Study, a nationally representative, longitudinal study of more than 46000 adults and youth participants in the US, using a four- stage stratified area probability sample design ${ }^{21}$. Data were collected using computer-assisted personal interviewing for screener and parent interviews, and audio computer assisted self-interviewing for adult and youth interviews. The weighted retention rate between Wave 1 and Wave 3 was 78.4\%. PATH collects information from youth and adults about use of various tobacco products including cigarettes and e-cigarettes, tobacco dependence, cessation, perceptions of risk and harm, overall physical and mental health, peer and family influences, substance use, and demographic information ${ }^{22}$. Information on the sampling procedures can be found in the PATH User Guide $^{21}$. For these analyses, the analytic sample consisted of 11801 youth aged $12-17$ years. Since the current study is a secondary data analysis of existing data, it is exempt from approval by the Institutional Review Board of the Johns Hopkins Bloomberg School of Public Health.

\section{Measures}

Current cigarette and e-cigarette use

In the PATH Study youth data, 'current use' of any tobacco product was defined as using a tobacco product within the past 30 days. If a participant used at least one other tobacco product (i.e. cigar, hookah, smokeless, snus, kretek) within the past 30 days, we coded them as 'other tobacco product users' (yes/no). A combined measure was created that categorized participants as follows: 1) dual users of cigarettes and e-cigarettes; 2 ) cigarette only users; 3 ) e-cigarette only users; 4) poly users (cigarette and e-cigarette users who use at least one other product); 5) cigarette and other product users; 6) e-cigarette and other product users; and 7) non-users of any tobacco product.

\section{Assessment of $A D H D$}

Parents of the youth were asked about their youth's ADHD history with the following question: 'In the past 12 months, has your child been told by a doctor, nurse or other health professional that (he/she) has ADHD?'. A follow-up question was asked to the parent of new baseline youth respondents who were not told they had ADHD in the past year: 'Has your child ever been told by a doctor, nurse or other health professional that (he/she) has ADHD or ADD?'. We coded 'ever ADHD diagnosis' as 'yes/no'. 


\section{Tobacco initiation age and quit attempts}

Tobacco initiation age was assessed by asking respondents the age ranges when they first started smoking cigarettes or e-cigarette use regularly with options 'aged 12-14 years' and 'aged 15-17 years'. Cigarette quit attempts for cigarette only users was assessed by 'In the past 12 months, have you tried to completely stop smoking cigarettes?' and e-cigarette quit attempts for e-cigarette only users was assessed by 'Have you tried to completely stop using [e-cigarette] within the past 12 months?'; both questions were coded as 'yes/no'.

\section{Covariates}

Participants were also assessed on their age (coded as $12-14$ years or 15-17 years); sex (coded as male; female); and race/ethnicity (coded as non-Hispanic white; non-Hispanic black; Hispanic; and others).

\section{Statistical analysis}

All analyses were performed using STATA version 15.1 (Statacorp., College Station, TX). The PATH Study population weights were used to adjust for the complex study design including oversampling and non-response. Fay's method, a variant of balanced repeated replication method, was used to form replicative weights in variance estimation in all analyses. The Fay coefficient was specified at 0.30, as recommended by the PATH Study ${ }^{20}$. We present unweighted frequencies and weighted percentages in the tables. Further information on the weighting procedure can be obtained from the PATH Study Public-Use Files ${ }^{17}$. Chi-squared, multivariable logistic regression, and multinomial logistic regression were used to examine the association between tobacco product use and ADHD status, controlling for covariates for potential confounding. All tests were two-sided with significance level set at $5 \%$.

\section{RESULTS}

The study population of 11801 youth included 5996 (50.4\%) aged $12-14$ years, 6095 (48.6\%) females, 5336 (53.1\%) non-Hispanic Whites, 132 (1.2\%) cigarette only users, $243(2.2 \%)$ e-cigarette only users, $87(0.8 \%)$ dual users of both cigarettes and e-cigarettes, $79(0.7 \%)$ cigarette and other product(s) users, $62(0.6 \%)$ e-cigarette and other product(s) users, $62(0.6 \%)$ poly users, and $10755(93.9 \%)$ non- users. The parents of $1240(10.8 \%)$ youth reported their child has ever been told by a health professional that he/she has ADHD (Table 1).

Among youth with ADHD, 18 (1.6\%) were cigarette only users, 30 (3.0\%) e-cigarette only users, 17 (1.7\%) dual users, $12(1.0 \%)$ cigarette and other product(s) users, $8(0.8 \%)$ e-cigarette and other product(s) users, 20 (1.6\%) poly users, and 1070 (90.3\%) nonusers. Among youth without ADHD, 114 (1.1\%) were cigarette only users, $213(2.1 \%)$ e-cigarette only users, $70(0.7 \%)$ dual users, $67(0.7 \%)$ cigarette and other product users, $54(0.6 \%)$ e-cigarette and other product(s) users, $42(0.5 \%)$ poly users, and 9685 $(94.3 \%)$ non-users $(\mathrm{p}<0.001)$ (Table 2$)$.

After adjustment for age, sex, and race/ethnicity, compared to youth without $\mathrm{ADHD}$, the relative

Table 1. Sociodemographic characteristics of participants, PATH study wave 3 (2015-2016) youth data, US (N=11801)

\begin{tabular}{|c|c|c|}
\hline Characteristics & $n$ & $\%(95 \%$ CI $)$ \\
\hline \multicolumn{3}{|l|}{ Age (years) } \\
\hline $12-14$ & 5996 & $50.4(49.9-50.9)$ \\
\hline $15-17$ & 5805 & $49.6(49.1-50.0)$ \\
\hline \multicolumn{3}{|l|}{ Sex } \\
\hline Male & 6095 & $51.4(51.2-51.6)$ \\
\hline Female & 5673 & $48.6(48.4-48.8)$ \\
\hline \multicolumn{3}{|l|}{ Race/ethnicity } \\
\hline Non-Hispanic White & 5336 & $53.1(52.7-53.5)$ \\
\hline Non-Hispanic Black & 1518 & $13.1(12.8-12.4)$ \\
\hline Hispanic & 3438 & $23.8(23.5-24.2)$ \\
\hline Other & 1098 & $9.9(9.7-10.3)$ \\
\hline \multicolumn{3}{|l|}{ Tobacco use status } \\
\hline Cigarette only users & 132 & $1.2(1.0-1.4)$ \\
\hline E-cigarette only users & 243 & $2.2(1.9-2.6)$ \\
\hline $\begin{array}{l}\text { Dual users of cigarettes and } \\
\text { e-cigarettes }\end{array}$ & 87 & $0.8(0.6-0.9)$ \\
\hline Cigarette and other product(s) users & 79 & $0.7(0.5-0.9)$ \\
\hline E-cigarette and other product(s) users & 62 & $0.6(0.5-0.8)$ \\
\hline $\begin{array}{l}\text { Poly users [cigarettes, e-cigarettes, } \\
\text { and other product(s)] }\end{array}$ & 62 & $0.6(0.5-0.8)$ \\
\hline Non-users & 10755 & $93.9(93.3-94.5)$ \\
\hline \multicolumn{3}{|l|}{ ADHD } \\
\hline No & 10561 & $10.8(10.0-11.5)$ \\
\hline Yes & 1240 & $89.2(88.5-90.0)$ \\
\hline Total & 11801 & 100.0 \\
\hline
\end{tabular}

ADHD: attention deficit hyperactivity disorder. 
Table 2. Tobacco use status of youth with and without ADHD and multinomial logistic regression model of being a tobacco user among youth with ADHD compared to youth without ADHD, PATH Study Wave 3 (20152016) Youth Data, US (N=10755)

\begin{tabular}{|c|c|c|c|c|c|c|}
\hline \multirow[t]{3}{*}{ Tobacco use status } & \multicolumn{4}{|c|}{$A D H D$} & \multirow[t]{3}{*}{$p^{*}$} & \multirow[t]{3}{*}{$\operatorname{RRR}^{a}(95 \% \mathrm{CI})$} \\
\hline & \multicolumn{2}{|c|}{ Yes } & \multicolumn{2}{|c|}{ No } & & \\
\hline & $n$ & $\%$ & $n$ & $\%$ & & \\
\hline Cigarette only users & 18 & 1.6 & 114 & 1.1 & & $1.79(1.02-3.21)$ \\
\hline E-cigarette only users & 30 & 3.0 & 213 & 2.1 & & $1.41(1.01-2.21)$ \\
\hline Dual users of cigarettes and e-cigarettes & 17 & 1.7 & 70 & 0.7 & & $3.40(1.69-6.84)$ \\
\hline Cigarette and other product(s) users & 12 & 1.0 & 67 & 0.7 & $<0.001$ & $1.75(0.92-3.35)$ \\
\hline E-cigarette and other product(s) users & 8 & 0.8 & 54 & 0.6 & & $1.48(0.58-3.77)$ \\
\hline Poly users [cigarettes, e-cigarettes, and other product(s)] & 20 & 1.6 & 42 & 0.5 & & $3.37(1.86-6.11)$ \\
\hline Non-users & 1070 & 90.3 & 9685 & 94.3 & & 1 \\
\hline
\end{tabular}

ADHD: attention deficit hyperactivity disorder. ${ }^{*}$ Chi-squared test. a Relative Risk Ratio: RRR was adjusted by age, sex, and race/ethnicity.

risk ratio (RRR) was 1.79 (95\% CI: 1.02-3.21) for cigarette only use, 1.41 (95\% CI: 1.01-2.21) for e-cigarette only use, 3.40 (95\% CI: 1.69-6.84) for dual use, 1.75 (95\% CI: 0.92-3.35) for cigarette and other product(s) use, 1.48 (95\% CI: 0.58-3.77) for e-cigarette and other product(s) use, and 3.37 (95\% CI: 1.88-6.17) for poly use among youth with ADHD (Table 2).

Among youth with ADHD, ever being a regular user of cigarettes and e-cigarettes before the age of 14 years was reported by $62.1 \%$ and $51.1 \%$ of youth, respectively; and $54.3 \%$ and $42.1 \%$ among youth without ADHD, respectively ( $\mathrm{p}=0.492$ and $\mathrm{p}=0.428)$. Among youth with ADHD and who currently used cigarettes or e-cigarettes, trying to quit cigarettes and e-cigarettes in the last 12 months was reported by $37.5 \%$ and $28.8 \%$, respectively, and by $40.7 \%$ and $28.3 \%$ of youth without ADHD, respectively $(p=0.736$ and $\mathrm{p}=0.934$ ) (data not shown).

With regard to ADHD prevalence, $13.7 \%$ of cigarette only users, $14.3 \%$ of e-cigarette only users, $23.7 \%$ of dual users of cigarettes and e-cigarettes, $15.6 \%$ of cigarette and other product(s) users, $14.0 \%$ of e-cigarette and other product(s) users, $26.7 \%$ of poly users, and $10.1 \%$ of non-users had ADHD diagnosis $(\mathrm{p}<0.001)$ (data not shown).

\section{DISCUSSION}

This is the first nationally representative study in the US to report higher e-cigarette use rates among youth with parent-reported ADHD compared to youth without ADHD. The e-cigarette use rate among youth with parent-reported ADHD was almost $50 \%$ higher than among youth without ADHD. In addition, poly use and dual use of both cigarettes and e-cigarettes were also higher among youth with ADHD compared to youth without ADHD. The association between ADHD and combustible cigarette use is wellestablished. This study extends this association to e-cigarettes that are very popular among today's youth in the US. The association between poly tobacco use and ADHD was also reported previously ${ }^{18}$, but not using nationally representative data. To the best of our knowledge, this is the first nationally representative study to report association between poly tobacco use and ADHD among youth. Consistent with the National Comorbidity Survey Replication study ${ }^{20}$, higher rates of e-cigarette use among youth whose parents report that they were diagnosed with ADHD is concerning. It is critical for healthcare providers to be screening youth for e-cigarette use, especially youth who are diagnosed with ADHD.

The mechanism of the association between ADHD and e-cigarette use still needs to be better understood. Several factors may contribute to the association observed in the current study. ADHD is characterized by difficulty in attention, impulsivity, and poor decision making ${ }^{5}$. All of these features and youth perceptions of e-cigarettes being less harmful than cigarettes ${ }^{23}$ may lead to higher e-cigarette use rates among youth with ADHD. In addition, in order to control their attentional dysfunction, some youth 
with ADHD may be self-medicating to alleviate their symptoms of ADHD by using nicotine ${ }^{7}$. It has been hypothesized that the greater incidence of smoking might reflect an attempt at self-medication of ADHD symptoms ${ }^{24}$ and indirect evidence from adults suggests that nicotine treatment can attenuate dysfunction among those with $\mathrm{ADHD}^{25,26}$. This theory, however, has not been sufficiently assessed among youth and the effect of tobacco use on ADHD symptoms among youth has not been studied thus far. Hence, it is not known whether tobacco use deteriorates or alleviates ADHD symptoms especially among youth.

ADHD is a risk factor for early onset of combustible tobacco use $\mathrm{e}^{14}$. In the current study, initiating regular use of e-cigarettes before the age of 14 years was almost $10 \%$ higher among youth with ADHD compared to youth without ADHD. This difference was not statistically significant, likely because of the low sample size; however, this outcome raises the concern that ADHD could also be a risk factor for early onset of e-cigarette use among youth in the US.

It is well known that people with ADHD have more difficulty quitting smoking ${ }^{17}$. In this study, the proportions of youth trying to quit e-cigarettes in the last 12 months were identical between youth with and without ADHD, consistent with the Pittsburgh ADHD Longitudinal Study (PALS) of youth and young adults ${ }^{27}$. However, young age, short usage time and small number of quit attempts of youth compared to adults could be an explanation of this result. In addition, given the low sample size in this study, more research is needed to understand the association between ADHD diagnosis and e-cigarette quit attempts among youth.

ADHD treatment has been found effective in decreasing the risk for smoking among youth ${ }^{28}$ but has not been found to be effective in improving smoking cessation success in adults ${ }^{29}$. Hence, treating ADHD during childhood or adolescence may be critical to help protect people with ADHD from becoming cigarette smokers in adulthood.

\section{Limitations}

This study has several limitations. First, the association between ADHD and tobacco use is cross-sectional. Therefore, it does not address the causal association between ADHD and e-cigarette use among youth.
Second, the ADHD diagnosis relied on parents' self-reports, though parents may be a more reliable reporter of their youth's health condition compared to their youth ${ }^{30}$. This is different from a number of studies examining ADHD and nicotine, which used a thorough diagnostic assessment of ADHD following DSM criteria. We do not know what (if any) diagnostic criteria were used by respondents, and it is likely that there are false positive and false negative cases. Other factors that were not assessed in the PATH study may also be associated with youth having ADHD symptoms, such as health insurance status. Third, we were not able to assess youth who had clinically nonsignificant ADHD symptoms; ENDS use rates might be higher among these youth compared to youth without ADHD symptoms. Fourth, we were unable to control for health insurance status of participants because having health insurance was not assessed by the PATH Study. Fifth, it is possible that internalizing/ externalizing problems may be related to e-cigarette use. Future work should consider examining these conditions while assessing the association between ADHD and e-cigarette use. Fifth, the information of tobacco use status of the participants relied on selfreported information and did not include biological verification of nicotine product use. Finally, there was a limited number of participants for assessing quit rates, initiation age for tobacco use, and taking ADHD medication regularly. Despite these limitations, relying on nationally representative data is a strength of this study.

\section{CONCLUSIONS}

This is the first nationally representative study to report that e-cigarette only use, dual use of both cigarettes and e-cigarettes, and poly use were significantly associated with parent report of an ADHD diagnosis among youth. Educating parents and youth about e-cigarettes, especially those with ADHD, may help to curb e-cigarette use among youth in the US. Further, it is critical for healthcare providers to be screening youth for e-cigarette use, especially youth who are diagnosed with ADHD. Further examination on the association between dual and poly tobacco use and ADHD is warranted, given the evolving landscape of available products and the paucity of published information on the health effects associated with different combinations of tobacco use. 


\section{REFERENCES}

1. Cullen KA, Gentzke AS, Sawdey MD, et al. e-Cigarette use among youth in the United States, 2019. JAMA. 2019;322(21):2095-2103. doi:10.1001/jama.2019.18387

2. Wang TW, Neff LJ, Park-Lee E, Ren C, Cullen KA, King BA. E-cigarette use among middle and high school students - United States, 2020. MMWR Morb Mortal Wkly Rep. 2020;69(37):1310-1312. doi:10.15585/mmwr.mm6937e1

3. U.S. Department of Health and Human Services. E-Cigarette use among youth and young adults: A report of the Surgeon General. U.S. Department of Health and Human Services, Centers for Disease Control and Prevention, National Center for Chronic Disease Prevention and Health Promotion, Office on Smoking and Health; 2016. Accessed June 20, 2020. https://www. cdc.gov/tobacco/data_statistics/sgr/e-cigarettes/index. htm\#report

4. Murthy VH. E-Cigarette Use Among Youth and Young Adults: A Major Public Health Concern. JAMA Pediatr. 2017;171(3):209-210. doi:10.1001/jamapediatrics.2016.4662

5. American Psychiatric Association. Diagnostic and statistical manual of mental disorders. 5th ed. American Psychiatric Association; 2013. doi:10.1176/appi.books.9780890425596

6. Kollins SH, McClernon FJ, Fuemmeler BF. Association between smoking and attention-deficit/hyperactivity disorder symptoms in a population-based sample of young adults. Arch Gen Psychiatry. 2005;62(10):11421147. doi:10.1001/archpsyc.62.10.1142

7. Gehricke JG, Loughlin SE, Whalen CK, et al. Smoking to self-medicate attentional and emotional dysfunctions. Nicotine Tob Res. 2007;9 Suppl 4:S523-S536. doi:10.1080/14622200701685039

8. Bekker EM, Böcker KBE, Van Hunsel F, van den Berg MC, Kenemans JL. Acute effects of nicotine on attention and response inhibition. Pharmacol Biochem Behav. 2005;82(3):539-548. doi:10.1016/j.pbb.2005.10.009

9. Wilens TE, Vitulano M, Upadhyaya H, et al. Cigarette smoking associated with attention deficit hyperactivity disorder. J Pediatr. 2008;153(3):414-419. doi:10.1016/j.jpeds.2008.04.030

10. Tercyak KP, Lerman C, Audrain J. Association of attentiondeficit/hyperactivity disorder symptoms with levels of cigarette smoking in a community sample of adolescents. J Am Acad Child Adolesc Psychiatry. 2002;41(7):799805. doi:10.1097/00004583-200207000-00011

11. Sibley MH, Pelham WE, Molina BSG, et al. The role of early childhood ADHD and subsequent CD in the initiation and escalation of adolescent cigarette, alcohol, and marijuana use. J Abnorm Psychol. 2014;123(2):362374. doi:10.1037/a0036585

12. Lambert NM, Hartsough CS. Prospective study of tobacco smoking and substance dependencies among samples of ADHD and non-ADHD participants. J Learn Disabil. 1998;31(6):533-544. doi:10.1177/002221949803100603

13. Klein RG, Mannuzza S, Olazagasti MA, et al. Clinical and functional outcome of childhood attention-deficit/hyperactivity disorder 33 years later. Arch Gen Psychiatry. 2012;69(12):12951303. doi:10.1001/archgenpsychiatry.2012.271

14. Milberger S, Biederman J, Faraone SV, Chen L, Jones J. ADHD is associated with early initiation of cigarette smoking in children and adolescents. J Am Acad Child Adolesc Psychiatry. 1997;36(1):37-44. doi:10.1097/00004583-199701000-00015

15. Fuemmeler BF, Kollins SH, McClernon FJ. Attention deficit hyperactivity disorder symptoms predict nicotine dependence and progression to regular smoking from adolescence to young adulthood. J Pediatr Psychol. 2007;32(10):1203-1213. doi:10.1093/jpepsy/jsm051

16. Pomerleau OF, Downey KK, Stelson FW, Pomerleau CS. Cigarette smoking in adult patients diagnosed with attention deficit hyperactivity disorder. J Subst Abuse. 1995;7(3):373-378. doi:10.1016/0899-3289(95)90030-6

17. Biederman J, Monuteaux MC, Mick E, et al. Is Cigarette Smoking a Gateway to Alcohol and Illicit Drug Use Disorders? A Study of Youths with and without Attention Deficit Hyperactivity Disorder. Biol Psychiatry. 2006;59(3):258-264. doi:10.1016/j.biopsych.2005.07.009

18. Goldenson NI, Khoddam R, Stone MD, Leventhal AM. Associations of ADHD symptoms with smoking and alternative tobacco product use initiation during adolescence. J Pediatr Psychol. 2018;43(6):613-624. doi:10.1093/jpepsy/jsx153

19. Dvorsky MR, Langberg JM. Cigarette and e-cigarette use and social perceptions over the transition to college: The role of ADHD symptoms. Psychol Addict Behav. 2019;33(3):318-330. doi:10.1037/adb0000450

20. Glantz MD, Anthony JC, Berglund PA, et al. Mental disorders as risk factors for later substance dependence: estimates of optimal prevention and treatment benefits. Psychol Med. 2009;39(8):1365-1377. doi:10.1017/S0033291708004510

21. United States Department of Health and Human Services. National Institutes of Health. National Institute on Drug Abuse, and United States Department of Health and Human Services. Food and Drug Administration. Center for Tobacco Products. Population Assessment of Tobacco and Health (PATH) Study [United States] Public-Use Files (ICPSR 36498). October 21, 2020. doi:10.3886/ICPSR36498.v11

22. Hyland A, Ambrose BK, Conway KP, et al. Design and methods of the Population Assessment of Tobacco and Health (PATH) Study. Tob Control. 2017;26(4):371-378. doi:10.1136/tobaccocontrol-2016-052934

23. Camenga DR, Cavallo DA, Kong G, et al. Adolescents' and Young Adults' Perceptions of Electronic Cigarettes for Smoking Cessation: A Focus Group Study. Nicotine Tob Res. 2015;17(10):1235-1241. doi:10.1093/ntr/ntv020

24. Levin ED, Conners CK, Sparrow E, et al. Nicotine effects on adults with attention-deficit/hyperactivity disorder. Psychopharmacology (Berl). 1996;123(1):55-63. 
doi:10.1007/BF02246281

25. Levin ED, Conners CK, Silva D, Canu W, March J. Effects of chronic nicotine and methylphenidate in adults with attention deficit/hyperactivity disorder. Exp Clin Psychopharmacol. 2001;9(1):83-90. doi:10.1037/1064-1297.9.1.83

26. Kollins SH, Sweitzer MM, McClernon FJ, Perkins KA. Increased subjective and reinforcing effects of initial nicotine exposure in young adults with attention deficit hyperactivity disorder (ADHD) compared to matched peers: results from an experimental model of first-time tobacco use. Neuropsychopharmacology. 2020;45(5):851856. doi:10.1038/s41386-019-0581-7

27. Rhodes JD, Pelham W, Gnagy EM, Shiffman S, Derefinko KJ, Molina BSG. Cigarette smoking and ADHD: An examination of prognostically relevant smoking behaviors among adolescents and young adults. Psychol Addict Behav. 2016;30(5):588-600. doi:10.1037/adb0000188

28. Hammerness P, Joshi G, Doyle R, et al. Do stimulants reduce the risk for cigarette smoking in youth with attention-deficit hyperactivity disorder? A prospective, long-term, open-label study of extended-release methylphenidate. J Pediatr. 2013;162(1):22-27.e2. doi:10.1016/j.jpeds.2012.06.046

29. Winhusen TM, Somoza EC, Brigham GS, et al. Does treatment of Attention Deficit Hyperactivity Disorder (ADHD) enhance response to smoking cessation intervention in ADHD smokers? A randomized trial. J Clin Psychiatry. 2010;71(12):1680-1688. doi:10.4088/JCP.09m05089gry

30. De Los Reyes A, Youngstrom EA, Swan AJ, Youngstrom JK, Feeny NC, Findling RL. Informant Discrepancies in Clinical Reports of Youths and Interviewers' Impressions of the Reliability of Informants. J Child Adolesc Psychopharmacol. 2011;21(5):417-424. doi:10.1089/cap.2011.0011

\section{CONFLICTS OF INTEREST}

The authors have each completed and submitted an ICMJE form for disclosure of potential conflicts of interest. The authors declare that they have no competing interests, financial or otherwise, related to the current work. B. Kaplan and J.E. Cohen report that they received support from the National Institute on Drug Abuse of the National Institutes of Health and the Center for Tobacco Products of the U.S. Food and Drug Administration (U54DA036105). A.V. Marcell reports that in the past 36 months he received grants from NIH (R21 HD097453) and BCHD Title $X$, Teen pregnancy prevention grant Boston Public School System Y2CONNECT.org

\section{FUNDING}

This research was supported by the National Institute on Drug Abuse of the National Institutes of Health under Award Number U54DA036105 and the Center for Tobacco Products of the U.S. Food and Drug Administration. The content is solely the responsibility of the authors and does not necessarily represent the views of the NIH or the FDA.

\section{ETHICAL APPROVAL AND INFORMED CONSENT}

This study used publicly available youth data from wave 3 (October 2015-2016) of the Population Assessment of Tobacco and Health (PATH) Study, a nationally representative, longitudinal study. Since the current study is a secondary data analysis of existing data, it is exempt from approval by the Institutional Review Board of the Johns Hopkins Bloomberg School of Public Health.

PROVENANCE AND PEER REVIEW

Not commissioned; externally peer reviewed. 